\title{
Aplicación de algoritmo de filtrado colaborativo para realizar recomendaciones de productos turísticos en el Estado de Oaxaca
}

\section{Appliance of collaborative filtering algorithm to make recommendations in the State of Oaxaca for touristic products}

\author{
HERNANDEZ-ABREGO, Anayansi Cristina $\dagger^{*}, \quad$ ALTAMIRANO-CABRERA, Marisol, \\ MATADAMAS-ORTIZ, Idarh Claudio y NEGRETE-PÉREZ, Maricela
}

Instituto Tecnológico de Oaxaca / Tecnológico Nacional de México-Departamento de Sistemas y Computación

ID $1^{\text {er }}$ Autor: Anayansi Cristina, Hernandez-Abrego / ORC ID: 0000-0002-8882-4842, CVU CONACYT ID: 748036

ID $1^{\mathrm{er}}$ Coautor: Marisol, Altamirano-Cabrera / ORC ID: 0000-0001-5800-9655, CVU CONACYT ID: 657390

ID $2^{\text {do }}$ Coautor: Idarh Claudio, Matadamas-Ortiz / ORC ID: 0000-0001-9358, CVU CONACYT ID: 447336

ID $3^{\text {er }}$ Coautor: Maricela, Negrete-Pérez / ORC ID: 0000-0001-5393

DOI: $10.35429 /$ JCA.2019.12.3.18.28

Recibido 20, 2019; Aceptado Junio 30, 2019

\begin{abstract}
Resumen
Oaxaca es un estado al que visitan millones de turistas al año, de acuerdo a datos de la Secretaría de Turismo en el año 2018, se tuvo una ocupación hotelera de 5,038,769 visitantes tanto nacionales como extranjeros. La cantidad de sitios que pueden visitar, rutas que pueden recorrer o actividades que pueden realizar, son muy variadas y tomar una decisión puede volverse complicado. Hay diversos sitios promocionales, los cuales pueden orientar al turista respecto de su viaje, sin embargo, lo que es agradable para uno, puede no serlo para otro, debido a que los perfiles de turistas son variados, dependiendo del objetivo de su visita, la edad que tenga, la cantidad de dinero que tiene destinado para el viaje, entre otras cosas. Poder predecir los productos turísticos que puedan satisfacer a los visitantes para mejorar su experiencia de viaje, es relevante tanto para el gobierno, los proveedores de servicios y por supuesto para los mismos turistas, por ello la importancia de aplicar un algoritmo de filtrado colaborativo para realizar recomendaciones a los turistas de sitios, hoteles y restaurantes que podrían agradarles y puntearlos en un mapa para su rápida referencia y localización.
\end{abstract}

Filtrado colaborativo, Algoritmo, Turismo

\begin{abstract}
The state of Oaxaca,México, is visited by millions of tourists every year. According to data from the Ministry of Tourism in 2018, there was a hotel occupancy of $5,038,769$ visitors, both locals and foreigners. The number of sites that can be visited, routes that can be toured or activities that can be done are varied so the making of decision process could become complicated. There are several promotional web sites that can guide tourists about their trip, however, what is nice for one, may not be for another, because the profiles of tourists vary depending on the purpose of their visit, their age, the amount of money they have destined for the trip, among other things. Being able to predict tourists products to improve their travel experience is very important for the government, service providers and of course, for tourists, therefore the importance of applying a collaborative filtering algorithm to make recommendations to the tourists about places, hotels and restaurants that could improve economy in the whole state. Besides, the algorithm could help to point the routes in a map for quick reference and location.
\end{abstract}

Collaborative filtering, Algorithm, Tourism

Citación: HERNANDEZ-ABREGO, Anayansi Cristina, ALTAMIRANO-CABRERA, Marisol, MATADAMAS-ORTIZ, Idarh Claudio y NEGRETE-PÉREZ, Maricela. Aplicación de algoritmo de filtrado colaborativo para realizar recomendaciones de productos turísticos en el Estado de Oaxaca. Revista de Cómputo Aplicado. 2019, 3-12: 18-28

\footnotetext{
$\dagger$ Investigador contribuyendo como primer Autor.

* Correspondencia del Autor (anayansi.ito@gmail.com)
} 


\section{Introducción}

El estado de Oaxaca se encuentra ubicado al suroeste de la República Mexicana, dividido en 8 regiones, es una entidad con gran riqueza natural y cultural, cuenta con más de 16 grupos étnicos. Su capital, la ciudad de Oaxaca de Juárez, se encuentra ubicada en la región de Valles Centrales, reconocida como patrimonio mundial de la humanidad; en el mes de julio del año 2019, la revista especializada Travel+Leisure ( 2019) la ubicó como la quinta mejor ciudad del mundo para viajar y la tercera en México.

La ciudad de Oaxaca, de acuerdo a datos de la Secretaría de Turismo (SECTUR,2018) recibe anualmente 1,349,72 turistas es decir, un promedio de 112,477 personas cada mes. Los motivos por los cuales estos turistas visitan la ciudad de Oaxaca son variados, así como la cantidad de dinero que están dispuestos a gastar, las actividades que les gustan y la cantidad de personas que los acompañan. Para los turistas buscar y seleccionar los restaurantes, hoteles y actividades entre los cientos que existen, sin tener previo conocimiento de esos lugares, no es sencillo.

Las reseñas y comentarios que dejan otros visitantes en redes sociales o en páginas oficiales, los pueden orientar, sin embargo, lo que es bueno para un perfil de turista, no necesariamente lo es para otro. Un sistema de recomendaciones, basado en la experiencia de visitantes con el mismo perfil puede ser de gran ayuda. Este artículo muestra la implementación de un algoritmo de filtrado colaborativo para realizar recomendaciones de productos turísticos en el estado de Oaxaca, el cual brinda un listado de lugares a visitar, acorde al perfil del turista.

Los sistemas de recomendación estudian las preferencias y gustos de los usuarios con el objetivo de recomendarles ítems a comprar o examinar los que factiblemente sean de su interés. (Ramovecchi, H. \& García, M., 2016)

Los sistemas de recomendación, como todos los sistemas de inteligencia artificial, se alimentan de datos. El hecho de que hoy la IA sea más tangible, se debe en gran medida a que cada interacción en las redes sociales, en la web o en los sistemas de mensajería aportan datos que permiten conocer gustos, tendencias, preferencias, etc.
Un sistema de recomendación tiene dos objetivos principales:

- Sugerir ítems en los que el usuario se espera que esté interesado, $\mathrm{y}$

Predecir la utilidad de un ítem específico para un usuario en particular.

Este es el primer sistema de recomendación de productos turísticos basado en un algoritmo de filtrado colaborativo para el turismo de Oaxaca.

Este artículo se ha dividido en 5 secciones, turismo, sistema de recomendación, metodología a desarrollar, resultados y conclusiones.

En la primera, turismo, se abordan los conceptos del sistema turístico y los tipos de turismo que podemos encontrar.

En la segunda sección, sistema de recomendación, se muestran las características de un sistema de recomendación, las diferentes técnicas de recomendación que existen.

En la tercera sección, metodología a desarrollar, se explica la metodología de desarrollo utilizada, sus características y cada una de sus fases.

En la cuarta sección, se muestran los resultados obtenidos una vez finalizado el sistema, el cual cumple con el objetivo planteado al inicio. conclusiones.

Y por último, se muestran las

\section{Turismo}

El turismo es un fenómeno social, cultural y económico relacionado con el movimiento de las personas a lugares que se encuentran fuera de su lugar de residencia habitual, por motivos personales o de negocios/profesionales. Estas personas se denominan visitantes y el turismo tiene que ver con sus actividades, de las cuales algunas implican un gasto turístico. (Morillo Moreno, 2011) 
La Organizacion Mundial del Turismo define al turismo como las actividades que realizan las personas durante sus viajes y estancias en lugares distintos al de su entorno habitual, por un período de tiempo consecutivo inferior a un año con fines de ocio, por negocios y otros. (OMT, 2007)

\section{El sistema turístico}

La naturaleza de la actividad turística es un resultado complejo de interrelaciones entre diferentes factores que hay que considerar conjuntamente desde una óptica sistemática, es decir, un conjunto de elementos interrelacionados entre sí que evolucionan dinámicamente. Concretamente, se distinguen cuatro elementos básicos en el concepto de actividad turística:

La demanda: formada por el conjunto de consumidores o posibles consumidores de bienes y servicios turísticos. (Organizacion Mundial del Turismo, 2011)

La oferta: compuesta por el conjunto de productos, servicios y organizaciones involucrados activamente en la experiencia turística. (Organizacion Mundial del Turismo, 2011)

El espacio geográfico: base física donde tiene lugar la conjunción o encuentro entre la oferta y la demanda y en donde se sitúa la población residente, que, si bien no es en sí misma un elemento turístico, se considera un importante factor de cohesión o disgregación, según se la haya tenido en cuenta o no a la hora de planificar la actividad turística. (Organizacion Mundial del Turismo, 2011)

Los operadores del mercado: son aquellas empresas y organismos cuya función principal es facilitar la interrelación entre la oferta y la demanda. Dentro de éstos se consideran las agencias de viajes, las compañías de transporte regular y aquellos organismos públicos y privados que, mediante su labor profesional, son artífices de la ordenación y/o promoción del turismo. (Organizacion Mundial del Turismo, 2011)

\section{Demanda turística}

Un concepto más estrictamente económico de estas magnitudes es la capacidad de gasto $-\mathrm{O}$ el gasto real de esas personas-, ya que en términos económicos la demanda es siempre una magnitud monetaria y no el número de consumidores. En este sentido, la OMT (1979) en sus definiciones enmarca el concepto de gasto por turismo, el cual define como "el gasto realizado por un visitante o de parte de un visitante por causa de un viaje y durante su estancia en el destino". (Gurría Di-Bella, 1991)

Sancho, citado por Navarro(2015), define que turistas, viajeros y visitantes forman la demanda turística $\mathrm{y}$, tras este término, se encuentra un grupo heterogéneo de personas, un agregado de personalidades e intereses con diferentes características sociodemográficas, motivaciones y experiencias. Por ello, son varias las clasificaciones que existen dentro de la demanda turística.

\section{Turista}

Las formas y estilos de vida y los atributos individuales de las personas -actitudes, percepciones, personalidad, experiencias $y$ motivaciones-, dan lugar a diferentes tipos de turistas que Cohen (1974) clasifica en cuatro grandes grupos, describiendo los dos primeros como turismo institucionalizado y los dos siguiente como turismo no institucionalizado. De acuerdo a Sancho, citado por Navarro(2015):

1. 1.El turista de masas organizado: tiene poco contacto con la cultura local y busca mantener su entorno habitual en el destino de vacaciones.

2. 2.El turista de masas individual: similar al anterior, aunque con un poco más de flexibilidad en sus decisiones.

3. 3.El explorador: organiza su viaje independientemente, aunque buscando transporte y alojamiento confortables. Abandona el entorno habitual, pero de vez en cuando vuelve a él.

4. 4.El impulsivo: no tiene ningún contacto con la industria turística, intenta abandonar su entorno de siempre, no tiene itinerario fijo y tiene contacto con las culturas que visita. 


\section{Oferta Turística}

El análisis económico entiende por oferta la cantidad de mercancía o servicio que entra en el mercado consumidor a un precio dado y por un periodo dado. En el caso de los servicios, cada vez que expira su tiempo de duración, su oferta se renueva, y si no se vende, se pierde. Para que un servicio turístico se convierta en oferta turística, es imprescindible que el consumidor potencial conozca su existencia. El turista real consumidor potencial es quien, localmente, establece el tiempo que cada producto puede entrar en el mercado. (Ibañez Pérez, Reyna \& Cabrera Villa, Carmelina (2011)

\section{Tipos de turismo}

La actividad turística se clasifica de acuerdo al componente espacial, temporal o incluso de acuerdo al propósito del viaje. Cada modalidad turística engloba un grupo amplio de actividades (Ibáñez \& Rodríguez Villalobos, 2012):

Turismo tradicional. Se le reconoce por ser masivo y el desarrollo de grandes instalaciones de alojamiento y espaciamiento. Regionalmente se caracteriza por las visitas a playa o a ciudades coloniales con atractivo culturales en grandes ciudades. (Ibáñez \& Rodríguez Villalobos, 2012)

Turismo alternativo. Esta corriente del turismo tiene por objetivo, la realización de viajes, en donde los visitantes participan en actividades recreativas de contacto directo con la naturaleza y las expresiones culturales de comunidades rurales, indígenas y urbanas, siendo respetuosos del patrimonio natural, cultural, e histórico del lugar que vistan. (Ibáñez \& Rodríguez Villalobos, 2012)

Esta otra clasificación agrupa actividades de acuerdo a las tendencias de cambio en las necesidades, gustos y preferencias de algunas personas o turistas:

Turismo cultural: "Aquel viaje turístico motivado por conocer, comprender y disfrutar el conjunto de rasgos y elementos distintivos, espirituales y materiales, intelectuales y afectivos que caracterizan a una sociedad o grupo social de un destino específico”.
En el caso de México el turismo cultural juega un papel muy importante para dar a conocer, preservar y disfrutar el patrimonio cultural y turístico, ya que el tratamiento adecuado del turismo cultural ha generado, desde una perspectiva de mercados, la satisfacción del cliente, la conservación del patrimonio de uso turístico y el desarrollo económico y social de las comunidades a partir de la generación de nuevos empleos. (Ibañez Pérez, Reyna \& Cabrera Villa, Carmelina, 2011)

Turismo religioso: son desplazamientos de personas por motivos de fervor y devoción religiosa, no importando el sexo, credo o estatus social, ya sea con la finalidad de cumplir un voto o una promesa, pedir algún tipo de beneficio o dar gracias por algún beneficio ya recibido. A diferencia del turismo tradicional, este tipo de turismo hace visitas por ocasiones repetidas al mismo sitio en un periodo menor y, por tanto, resulta más fiel al sitio que visita. (Ibañez Pérez, Reyna \& Cabrera Villa, Carmelina, 2011).

Turismo gastronómico: quienes visitan un destino en particular sólo para conocer y experimentar determinados tipos de comidas y bebidas particulares de la región, para conocer las especialidades gastronómicas que se preparan con motivos o en fechas específicas que coinciden con fiestas cívicas o culturales de importancia local y/o nacional, o porque son de temporada, para conocer sus ingredientes e incluso para aprender a prepararlos, es decir, para descubrir nuevas sensaciones y experiencias culinarias. (Ibañez Pérez, Reyna \& Cabrera Villa, Carmelina, 2011)

Turismo idiomático: desplazamiento cuya motivación principal es aprender un idioma; en este sentido, el español, inglés, francés y japonés son recursos turísticos con un gran potencial, ya que son idiomas que cada vez adquieren mayor relevancia socioeconómica y se están convirtiendo en una herramienta indispensable para maestros, empresarios, diplomáticos, universitarios, científicos y políticos. (Ibañez Pérez, Reyna \& Cabrera Villa, Carmelina, 2011)

Turismo de salud: se refiere a las técnicas existentes para el tratamiento de enfermedades o simple relajación en spas, saunas u otros centros de medicina tradicional o alternativa. 
En países como el nuestro esta actividad genera una derrama importante de divisas, e incluso la permanencia de este tipo de turistas puede ser más prolongada y generar mayor derrama que turistas que vienen de negocios. (Ibañez Pérez, Reyna \& Cabrera Villa, Carmelina, 2011)

Turismo de activo: está representado por el conjunto de turistas que realizan viajes relacionados con la práctica de algún deporte, desde el más barato y común, hasta el más costoso y sofisticado. Buscan, además, realizar una actividad física que suponga un cambio en la rutina diaria. Por tanto, una característica más de este tipo de turismo es la demanda de actividades que les permitan romper con la rutina diaria, con la monotonía y el estrés. Pero, que, al mismo tiempo, les permitan realizar alguna actividad física.

Turismo de parques temáticos: el conjunto de turistas que viajan con la finalidad de disfrutar de una atracción o elemento destinado o encaminado al disfrute de las diversiones, entretenimientos y otros recursos de ocio, ya sean naturales o creadas por el hombre, especialmente para atraer visitantes. (Ibañez Pérez, Reyna \& Cabrera Villa, Carmelina, 2011)

Turista de negocios: el conjunto de corrientes turísticas cuyo motivo de viaje está vinculado con la realización de actividades laborales y profesionales. Puede ser realizado de manera grupal o individual e incluye numerosos segmentos y productos relacionados con la organización de reuniones de negocios, con diferentes propósitos y magnitudes; además, comprende a los viajeros de negocios, que por cuestiones inherentes a sus actividades se desplazan de una ciudad a otra o de un país a otro para desarrollar actividades relacionadas con su profesión, por ejemplo: congresos, convenciones, incentivos y exposiciones. (Ibañez Pérez, Reyna \& Cabrera Villa, Carmelina, 2011).

\section{Servicio Turístico}

Conjunto de actividades perfectamente diferenciadas entre sí, pero íntimamente relacionadas, que funcionan en forma armónica y coordinada con el objeto de responder a las exigencias de servicio planteadas por la composición socioeconómica de una determinada corriente turística. (Ramírez, 2012)
Toda actividad realizada por una persona física o moral, pública o privada, tendiente a satisfacer necesidades específicas directamente planteadas por el desplazamiento turístico. (Ramírez, 2012)

\section{Sistema de recomendación}

Un sistema de de recomendación se basa en que personas con gustos similares, puedan compartir su percepción del lugar visitado, ayudando a otras personas en la selección de lugares a visitar. Hoy en día Internet pone a sus usuarios tantas opciones que un simple buscador no es suficiente, pero a través de Inteligencia Aritificial o de Machine Learning, se pueden crear algoritmos que repliquen la lógica que sigue una persona antes de tomar una decisión, la cual lo lleva a preguntarle a uno o varios amigos, con los que comparta gustos o a un experto en el cual confíe. La diferencia es que actualmente a través de la tecnología, en lugar de preguntar a dos o tres personas, se equipararía a preguntarle a una ciudad completa.

Este tipo de sistemas, como todos los sistemas de inteligencia artifical, se alimentan de datos obtenidos de la interacción de los usuarios en diversos sistemas, redes sociales, sistemas de mensajería, entre otros, los cuales permiten conocer gustos, tendencias y preferencias.

A través de este tipo de sistemas, un usuario que realiza un tipo de búsqueda de lugares para hospedarse o visitar, ya no será una lista interminable, sino que se le mostrará únicamente una lista corta de opciones, basado en recomendaciones realizadas por usuarios con perfiles similares al suyo.

Sin embargo, no sólo existen ventajas en este tipo de sistemas de recomendación, se pueden filtrar opciones de manera errónea cuando se trata de productos turísticos nuevos o cuando tienen pocas puntuaciones, lo cual se conoce como "arranque en frío", además, al solicitar datos personales de los usuarios, se puede caer en problemas de privacidad (Pazzani, 1999) de los datos o en ocasiones, se le presentan al usuario formularios muy extensos para llenar, lo cual puede ocasionar desmotivación y falta de interés, abandonando definitivamente el sistema. Aunado a que el mantenimiento de este tipo de sistemas suelen ser costosos (Vargas \& Leiva,2016) por la cantidad de datos que alojan y la cantidad de usuario que se conectan a él.

HERNANDEZ-ABREGO, Anayansi Cristina, ALTAMIRANO CABRERA, Marisol, MATADAMAS-ORTIZ, Idarh Claudio y NEGRETE-PÉREZ, Maricela. Aplicación de algoritmo de filtrado colaborativo para realizar recomendaciones de productos turísticos en el Estado de Oaxaca. Revista de Cómputo Aplicado. 2019. 


\section{Técnicas de recomendación}

Aunque este artículo no pretente mostrar un análisis de las diferentes técnicas de recomendación existentes, es importante conocerlas para identificar la utilizada y sus características. De acuerdo a Vargas y Leiva(2019), en su artículo titulado Sistema de recomendación turístico grupal basado en personalidad, se identifican las siguientes técnicas:

1. Sistemas de recomendación colaborativos.

2. Sistemas de recomendación basados en contenidos.

3. Sistemas de recomendación demográficos.

4. Sistemas de recomendación basados en conocimiento.

5. Sistemas de recomendación contextuales.

6. Sistemas de recomendación híbridos.

7. Sistemas de recomendación grupales.

8. Sistemas de recomendación basados en personalidad.

Los sistemas de recomendación colaborativo, es una de los más extendidos, es utlizado por empresas como Spotify. La técnica que utilizan este tipo de sistemas se basa en valoraciones que los usuarios hacen a productos, identificando sus gustos o perfil, para posteriormente hacer una recomendación de esos productos a personas con gustos similares.

\section{Sistema de recomendación en Oaxaca}

Actualmente no existe un sitio de recomendación en el estado de Oaxaca con estas características. Este sistema de recomendación pretende que el gobierno del estado de Oaxaca a través de la Secretaría de Turismo, en coordinación con los prestadores de servicios, mejoren la experiencia de viaje de los turistas, lo cual permita que regresen y recomienden los lugares que visitaron.
Fortalecer la actividad turística requiere del conocimiento de la percepción que los visitantes y turistas tienen de los servicios utilizados, para ello se requiere de un sistema de puntuación, en el cual puedan calificar dicho servicio a través de una escala que va del 1 al 7 , así como conocer otras característica del visitante como su edad, sexo, tipo de turismo que le gusta realizar, motivo del viaje, modo de viaje, tiempo estimado de duración de la visita y el presupuesto que tiene considerando para gastar durante su visita.

Por otro lado, un registro de los lugares que prestan servicio al turismo como hoteles, hostales, restaurantes, museos, etc, indicando además del nombre, su dirección e información adicional, en el caso de los lugares de alojamiento la cantidad de estrellas y el costo. De los restaurantes el tipo de comida que ofrecen y así de cada uno de los tipos de servicios que utilizan los visitantes y turistas. Con estos datos, se puede aplicar la técnica de recomentación colaborativa.

\section{Metodología a desarrollar}

Para el desarrollo del sistema de recomendación de filtrado colaborativo de productos turísticos del Estado de Oaxaca, se utilizó una metodología de desarrollo XP, ésta es una Metodología ligera de desarrollo de aplicaciones que se basa en la simplicidad, la comunicación y la realimentación del código desarrollado. (Universidad Nacional Autónoma de Nicaragua, Managua, 2016)

\section{Fases de la programación extrema (XP)}

Acorde con Roger Pressman (2010), "La programación extrema usa un enfoque orientado a objetos como paradigma preferido de desarrollo, y engloba un conjunto de reglas y prácticas que ocurren en el contexto de cuatro actividades estructurales: planeación, diseño, codificación y pruebas".

\section{Planeación}

La Metodología XP plantea la planificación como un diálogo continuo entre las partes involucradas en el proyecto, incluyendo al cliente, a los programadores y a los coordinadores. El proyecto comienza recopilando las historias de usuarios. 
Una vez obtenidas estas historias de usuarios, los programadores evalúan rápidamente el tiempo de desarrollo de cada una. (S. Pressman, 2010) Los Conceptos básicos de la planificación son:

- $\quad$ Las Historias de Usuarios, las cuales son descritas por el cliente.

- El Plan de Entregas (Release Plan), establece que las historias de usuarios serán agrupadas para conformar una entrega y el orden de las mismas.

Plan de Iteraciones (Iteration Plan), las historias de usuarios seleccionadas para cada entrega son desarrolladas y probadas en un ciclo de iteración, de acuerdo al orden preestablecido.

Reuniones Diarias de Seguimiento (Stand - Up Meeting), el objetivo es mantener la comunicación entre el equipo $\mathrm{y}$ compartir problemas $\mathrm{y}$ soluciones.

\section{Diseño}

El diseño XP sigue rigurosamente el principio MS (mantenlo sencillo). Un diseño sencillo siempre se prefiere sobre una representación más compleja. Además, el diseño guía la implementación de una historia conforme se escribe: nada más y nada menos. Se desalienta el diseño de funcionalidad adicional porque el desarrollador supone que se requerirá después. Los conceptos más importantes de diseño en esta metodología son los siguientes: (S. Pressman, 2010)

- Simplicidad, Un diseño simple se implementa más rápidamente que uno complejo. Por ello XP propone implementar el diseño más simple posible que funcione. (S. Pressman, 2010)

- Soluciones "Spike", Cuando aparecen problemas técnicos, o cuando es difícil de estimar el tiempo para implementar una historia de usuario, pueden utilizarse pequeños programas de prueba (llamados "Spike"), para explorar diferentes soluciones. (S. Pressman, 2010)
Recodificación ("Refactoring"),

Consiste en escribir nuevamente parte del código de un programa, sin cambiar su funcionalidad, a los efectos de crearlo más simple, conciso y entendible. Las metodologías de XP sugieren re codificar cada vez que sea necesario. (S. Pressman, 2010)

\begin{abstract}
Metáforas, XP sugiere utilizar este concepto como una manera sencilla de explicar el propósito del proyecto, así como guiar la estructura del mismo. Una buena metáfora debe ser fácil de comprender para el cliente y a su vez debe tener suficiente contenido como para que sirva de guía a la arquitectura del proyecto. (S. Pressman, 2010)
\end{abstract}

\section{Codificación}

Un concepto clave durante la actividad de codificación (y uno de los aspectos del que más se habla en la XP) es la programación por parejas. XP recomienda que dos personas trabajen juntas en una estación de trabajo con el objeto de crear código para una historia. Esto da un mecanismo para la solución de problemas en tiempo real (es frecuente que dos cabezas piensen más que una) y para el aseguramiento de la calidad también en tiempo real (el código se revisa conforme se crea). (Joskowicz, 2008)

\section{Pruebas}

Pruebas Unitarias: todos los módulos deben de pasar las pruebas unitarias antes de ser liberados o publicados. (Joskowicz, 2008)

Detección y Corrección de Errores, cuando se encuentra un error ("Bug"), éste debe ser corregido inmediatamente, y se deben tener precauciones para que errores similares no vuelvan a ocurrir. Asimismo, se generan nuevas pruebas para verificar que el error haya sido resuelto. (Joskowicz, 2008)

Pruebas de Aceptación: son creadas en base a las historias de usuarios, en cada ciclo de la iteración del desarrollo. El Cliente debe especificar uno o diversos escenarios para comprobar que una historia de usuario ha sido correctamente implementada. Asimismo, en caso de que fallen varias pruebas, deben indicar el orden de prioridad de resolución. 
Una historia de usuario no se puede considerar terminada hasta que pase correctamente todas las pruebas de aceptación. (Joskowicz, 2008)

\section{Resultados}

Durante la fase de planeación del sistema, se obtuvo la lista maestra de historias de usuario y se determinó que el desarrollo se haría en tres iteraciones, como se muestra en la tabla 1.

\begin{tabular}{|l|l|r|}
\hline \multicolumn{1}{|c|}{ Nombre de Historia } & Prioridad & Iteración \\
\hline $\begin{array}{l}\text { Interfaz Acceso y registro a la } \\
\text { plataforma }\end{array}$ & Alta & 1 \\
\hline $\begin{array}{l}\text { Interfaz Visualización de } \\
\text { productos turísticos }\end{array}$ & Media & 1 \\
\hline $\begin{array}{l}\text { Interfaz Registro de productos } \\
\text { turísticos }\end{array}$ & Media & 1 \\
\hline $\begin{array}{l}\text { Interfaz Actualización de } \\
\text { productos turísticos }\end{array}$ & Media & 2 \\
\hline Visualización de productos & Alta & 2 \\
\hline $\begin{array}{l}\text { Búsquedas } \\
\text { recomendaciones de productos } \\
\text { turísticos }\end{array}$ & Alta & 2 \\
\hline Productos turísticos & Alta & 3 \\
\hline $\begin{array}{l}\text { Evaluación productos turísticos } \\
\text { recomendaciones }\end{array}$ & Alta & 2 \\
\hline Dashboard del administrador & \\
\hline
\end{tabular}

Tabla 1 Lista maestra de Historias de Usuario Fuente: Elaboración Propia

Se hizo una categorización de productos turísticos que se presentan en el sistema, la cual, permite agregar a nuevos proveedores $\mathrm{y}$ prestadores de servicios para ser evaluados por los turistas y a su vez, presentarlos en las recomendaciones.
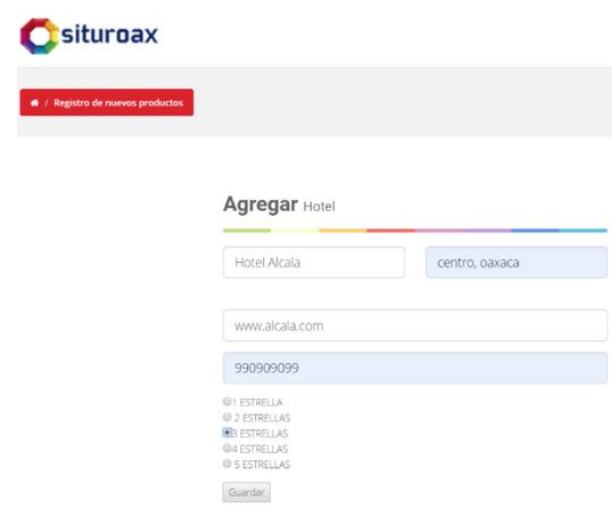

Figura 1 Agregar un nuevo proveedor de servicios Fuente: Elaboración Propia

Se mantuvo un diseño de interfaces gráficas de usuario sencillas, como la metodología lo indica.
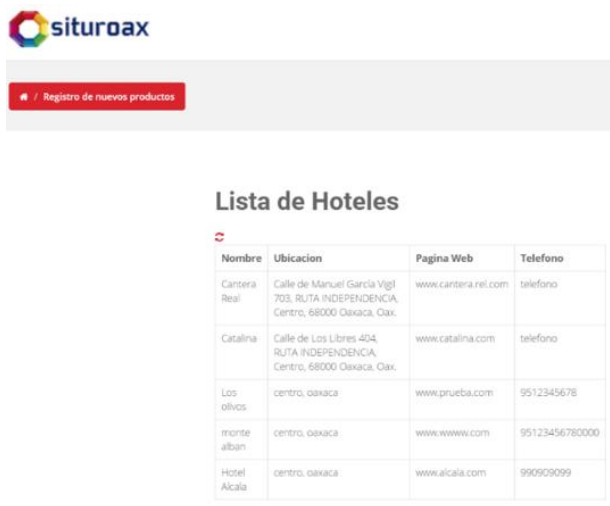

Figura 2 Listado de productos registrados Fuente: Elaboración Propia

Una vez finalizadas todas las fases de la primera iteración, se continuó con el desarrollo de las historias de usuario de la segunda iteración, correspondientes a la valoración de los lugares visitados y la búsqueda de recomendación.

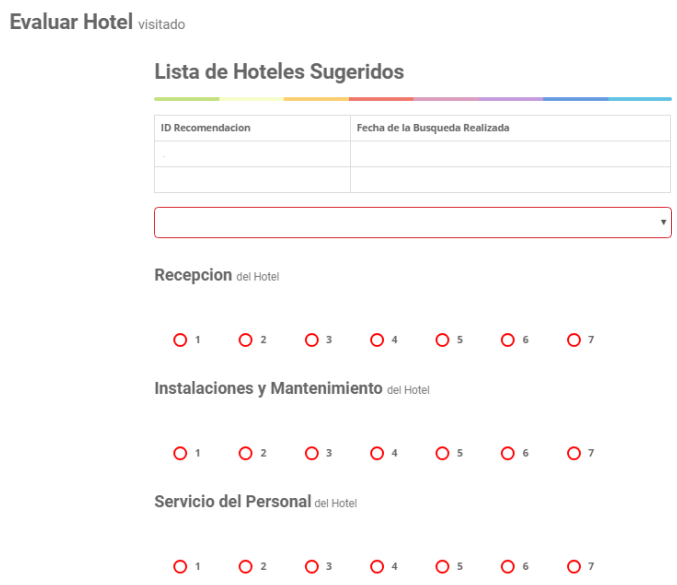

Figura 3 Interfaz de evaluación de productos turísticos Fuente: Elaboración Propia

Un usuario de servicios turísticos a través de una interfaz sencilla, puede evaluar varios aspectos del servicio brindado, utilizando una escala de 1 a 7.

Fue durante la segunda iteración que se implementó el algoritmo de filtrado colaborativo, utilizando el lenguaje R.

Para la búsqueda de productos turísticos, se creó una interfaz que recoge los datos del tipo de turismo, motivo de visita, modo de viaje, tiempo de su visita y presupuesto considerado para su permanencia. Esto con el fin de establecer un perfil de usuario para relacionarlos con otros usuarios. 


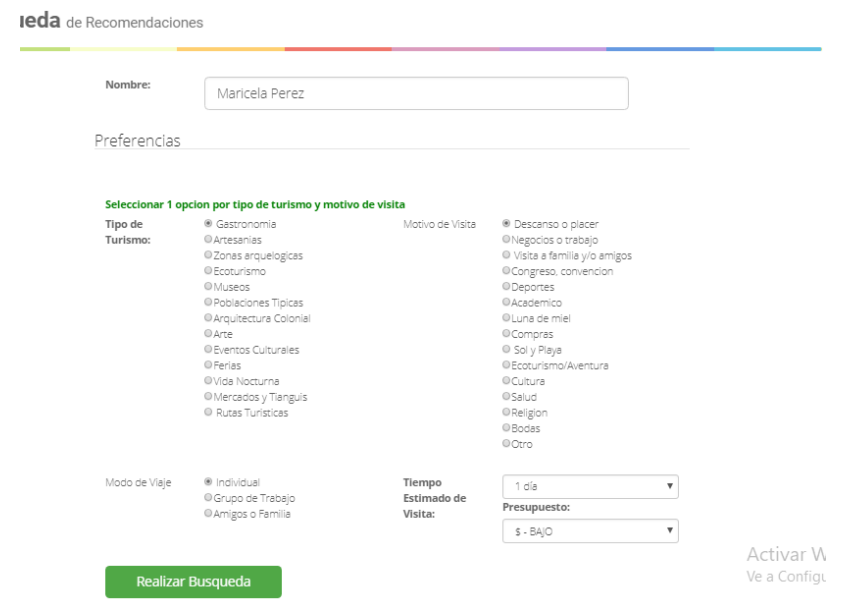

Figura 4 Interfaz de captura de preferencias del usuario Fuente: Elaboración Propia

Cuando el usuario presiona el botón "Realizar Búsqueda", el algoritmo se ejecuta con los parámetros seleccionados en el formulario de búsqueda. Considera el motivo de visita y el tipo de turismo del usuario para hacer la clasificación del perfil.

La matriz de calificaciones que maneja $\mathrm{R}$ para hacer la sugerencia considera los demás perfiles para obtener el resultado para el perfil del usuario.

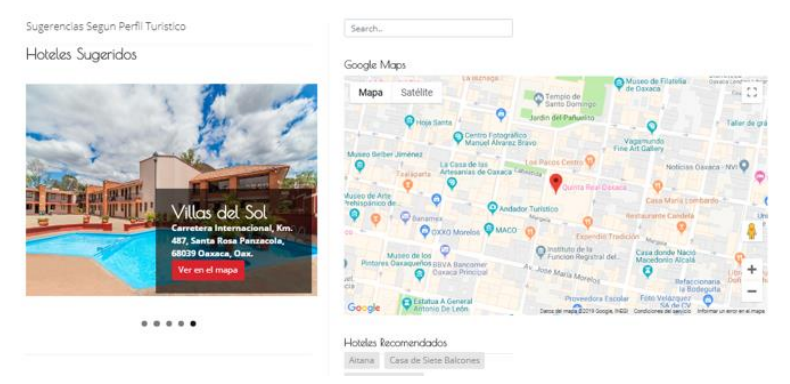

Figura 5 Interfaz de sugerencias para el usuario, resultado de la búsqueda

Fuente: Elaboración Propia

El sistema muestra una interfaz con la sugerencia de 5 lugares, mostrando los datos generales y su ubicación en un mapa.

El código de sugerencia, hace una búsqueda de todos los turistas que han visitado el tipo de producto seleccionado y lo han puntuado. Verifica cuántas valoraciones existen. Recorre todos los productos turísticos existentes del tipo seleccionado.

Si no hay un mínimo de usuarios que han visitado un producto turístico, no se considera una estimación suficientemente buena, por lo que lo elimina
Se obtienen los 10 usuarios más parecidos de entre los que han visitado el producto, cuya similitud es mayor o igual a $0 . \mathrm{Si}$ no hay un mínimo de usuarios con valoraciones válidas, no se considera una estimación suficientemente buena por lo que se pasa al siguiente producto.

Se obtienen las valoraciones de los 10 usuarios sobre el producto que se está evaluando. Se saca la media ponderada de las valoraciones de ellos. Se filtran los 5 productos con mayor puntuación de esos 10 usuarios, que son los que se muestran al usuario que realizó la búsqueda de sugerencias.

Para finalizar, en la tercera iteración se desarrolló un Dashboard que permite al administrador visualizar gráficamente la cantidad de búsquedas realizadas y el motivo de su visita.

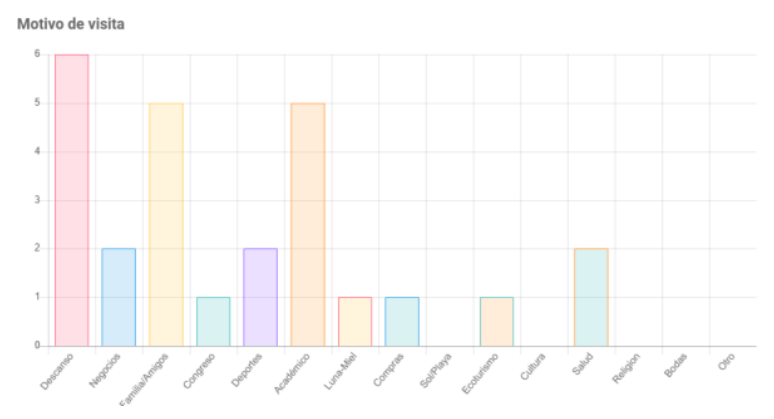

Figura 6 Dashboard Motivo de visita Fuente: Elaboración Propia

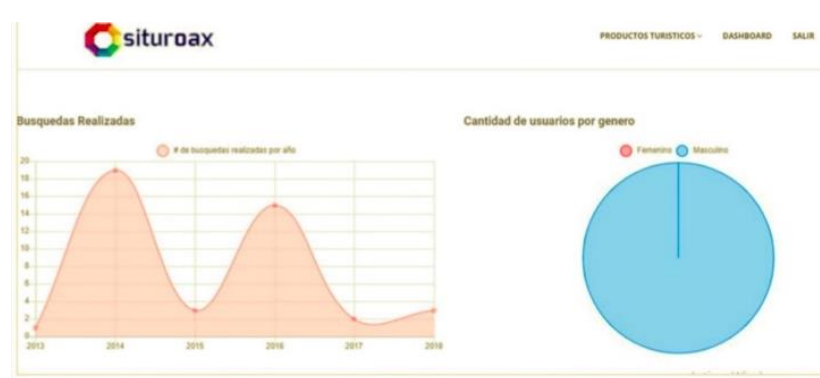

Figura 7 Dashboard Búsquedas registradas Fuente: Elaboración Propia

\section{Conclusiones}

Una vez finalizado el desarrollo del sistema, se obtuvo como resultado un sistema de puntuación y recomendación de productos turísticos para el estado de Oaxaca, basado en filtrado colaborativo. 
Este tipo de algoritmo aplicado para fortalecer la actividad turística de un lugar como Oaxaca, beneficia a turistas, prestadores de servicios turísticos y gobierno. Esta herramienta le permite a los turistas, tomar decisiones más acertadas respecto al lugar en el cual hospedarse, los restaurantes a los cuales ir y las actividades o lugares por visitar.

Por otro lado, los prestadores de servicios turísticos podrán conocer la percepción que tienen sus clientes del servicio recibido, para mejorar su servicio y sea ese mismo servicio el que los recomiende a otros usuarios. El gobierno se beneficia, porque la economía de los lugares visitados se reactiva, así como pueden tomar decisiones de qué zonas y municipios deben fortalecer y mejorar sus vías de comunicación y su derrama económica.

Este tipo de sistemas, mejoran conforme se utilizan, porque entre más datos y opiniones se tengan, las sugerencias serán más acertadas.

\section{Referencias}

Arteaga, J. (Junio de 2014). Estudio comparativo de metodologías de desarrollo de software [Tabla]. Recuperado de http://biblioteca.udenar.edu.co:8085/atenea/bibl ioteca/90255.pdf

Castellano,E. (2007). Evaluación del uso de algoritmos colaborativos para orientar académicamente al alumnado en bachillerato. (Memoria Investigadora del segundo año de Doctorado). Departamento de Informática, Universidad de Jaén, Jaén, España.

Contreras, J., Molina, E., \& Arteaga, P. (s.f.). Introducción a la programación estadística con $R$ para profesores. Mexico .

Facultat d'Informática de Barcelona. (s.f. de s.f. de s.f.). Intel.ligència Artificial.Barcelona, España. Recuperado de lsi.upc.es: http://www.lsi.upc.es/ bejar/ia/ia.html

Gurría, M. (1991). Introduccion al turismo. D.F., México: Editorial Trillas.

Hurwitz, J., \& Kirsch, D. (2018). Machine Learning For Dummies®, IBM Limited Edition. River St, Hoboken: John Wiley \& Sons, Inc.
Ibañez, R. \& Cabrera, C. (2011). Teoría General del Turismo: un enfoque global y nacional. Serie didáctica, UABCS. Baja California Sur, México. Recuperado de http://uabcs.mx/difusion2017/files/libros/pdf/18 4_20160908023838.pdf

Ibáñez, R. \& Rodríguez, I.(2012). Tipologías y antecedentes de la actividad turística: turismo tradicional y turismo alternativo. Fondo para la comunicación y la educación ambiental, A.C., México.Recuperado de https://agua.org.mx/wpcontent/uploads/2017/12/Tipologias-yantecedentes-de-la-actividad-turistica.pdf

Joskowicz, J. (2008). Reglas y Prácticas en eXtreme Programming. Montevideo,Uruguay. Recuperado https://iie.fing.edu.uy/ josej/docs/XP\%20\%20Jose\%20Joskowicz.pdf

Letelier, P. \& Penadés, M. (2006). Métodologías ágiles para el desarrollo de software: eXtreme Programming (XP). Técnica Administrativa. Recuperado de http://users.dsic.upv.es/asignaturas/eui/lds/doc/ masyxp.pdf

Morillo, M. (2011). Turismo y producto Turístico. Evolución, conceptos, componentes y clasificación. Visión Gerencial. ISSN: 13178822. Recuperado de https://www.redalyc.org/articulo.oa?id=465545 890011

Navarro, D. (2015). Recursos turísticos y atractivos turísticos: conceptualización, clasificación y valoración. Cuadernos de Turismo, ISSN: 1139-7861. Recuperado de http://www.redalyc.org/articulo.oa?id=398/398 38701014

OMT-Organización Mundial del Turismo(2007). Datos Esenciales del Turismo, Edición 2007. Recuperado de http:// www.unwto.org/infoshop

Orjuela, A., \& Rojas C., M. (02 de Junio de 2008). Las Metodologías de Desarrollo Ágil como una Oportunidad para la Ingeniería del Software Educativo. Recuperado de https://www.redalyc.org/pdf/1331/1331150270 22.pdf 
Pressman, R. (2010). Ingeniería del software. Un enfoque práctico. México: McGraw-Hill Interamericana editores, S.A. de C.V.

Ramírez, C. (2002).Calidad Total de las Empresas Turísticas (reimp.2012). D.F., México: Trillas.

Ramovecchi, H. \& - García, M. (2016). JoyMeter - Sistema de recomendación de actividades a usuarios de dispositivos móviles. 10 de agosto de 2019, de RIDAA UNICEN Sitio web: https://www.ridaa.unicen.edu.ar/xmlui/bitstrea $\mathrm{m} /$ handle/123456789/1346/Ramovecchi\%2c\%2 0Hernán\%20y\%20Garc\%C3\%ADa\%2c\%20Ma r\%C3\%ADa\%20Sol.PDF

Revista Travel + Leisure (julio, 2019). Las 5 mejores ciudades en México Recuperado de https://www.travelandleisure.com/worldsbest/cities-in-mexico

Revista Travel + Leisure (julio, 2019).15 mejores ciudades del mundo Recuperado de https://www.travelandleisure.com/worldsbest/cities

Rodrigo, J. (s.f. de Marzo de 2018). R Pubs, Sistemas de recomendación con R. Recuperado de https://rpubs.com/Joaquin_AR/370301

Romero, J., Dafonte, C., Gómez, Á., \& Penousal, F. J. (2007). Inteligencia Artificial y Computación Avanzada. Santiago de Compostela: Fundación Alfredo Brañas.

Secretaría de Turismo del Estado de Oaxaca (2018). Indicadores de la actividad turística 2018. Recuperado de http://www.sectur.oaxaca.gob.mx/wpcontent/uploads/2019/03/Actividad-turistica2018-cierre-web.pdf

Stanford University. (1998). Introduction to Machine Learning. Stanford, CA 94305: Robotics Laboratory, Department of Computer Science. Recuperado de https://ai.stanford.edu/ nilsson/MLBOOK.pdf

Universidad Nacional Autónoma de Nicaragua, Managua. (28 de Enero de 2016). Metodología Ágil de desarrollo de software programacion extrema. Recuperado de http://repositorio.unan.edu.ni/1365/1/62161.pdf
HERNANDEZ-ABREGO, Anayansi Cristina, ALTAMIRANOCABRERA, Marisol, MATADAMAS-ORTIZ, Idarh Claudio y NEGRETE-PÉREZ, Maricela. Aplicación de algoritmo de filtrado colaborativo para realizar recomendaciones de productos turísticos en el Estado de Oaxaca. Revista de Cómputo Aplicado. 2019. 